\title{
Compositional and functional properties of high-density lipoproteins in relation to coronary in-stent restenosis
}

Shiva Ganjali ${ }^{1}$, Elahe Mahdipour ${ }^{1}$, Seyed Hamid Aghaee-Bakhtiari², Majid Ghayour-Mobarhan ${ }^{3}$, Sara Saffar Soflaei ${ }^{3}$, Maciej Banach ${ }^{4,5}$, Anatol Kontush ${ }^{6}$, Amirhossein Sahebkar $r^{7,8,9,10}$

${ }^{1}$ Department of Medical Biotechnology and Nanotechnology, Faculty of Medicine, Mashhad University of Medical Sciences, Mashhad, Iran

${ }^{2}$ Bioinformatics Research Group, Mashhad University of Medical Sciences, Mashhad, Iran

${ }^{3}$ Department of Modern Science and Technology, Faculty of Medicine, Mashhad University of Medical Sciences, Mashhad, Iran \& UNESCO Higher Center, Nutrition Department, Mashhad University of Medical Sciences, Mashhad, Iran ${ }^{4}$ Department of Preventive Cardiology and Lipidology, Medical University of Lodz, Lodz, Poland

${ }^{5}$ Cardiovascular Research Centre, University of Zielona Gora, Zielona Gora, Poland ${ }^{6}$ National Institute for Health and Medical Research (INSERM), Research Unit 1166, Faculty of Medicine Pitié-Salpêtrière, Sorbonne University, Paris, France

${ }^{7}$ Biotechnology Research Center, Pharmaceutical Technology Institute, Mashhad University of Medical Sciences, Mashhad, Iran

${ }^{8}$ Applied Biomedical Research Center, Mashhad University of Medical Sciences, Mashhad, Iran

${ }^{9}$ School of Medicine, The University of Western Australia, Perth, Australia

${ }^{10}$ School of Pharmacy, Mashhad University of Medical Sciences, Mashhad, Iran

Submitted: 25 July 2021; Accepted: 2 August 2021

Online publication: 2 August 2021

Arch Med Sci 2023; 19 (1): 57-72

DOI: https://doi.org/10.5114/aoms/140728

Copyright $\odot 2021$ Termedia \& Banach

\section{Abstract}

Introduction: In-stent restenosis (ISR) is an unfavorable outcome that occurs in patients after coronary stenting. Use of drugs such as statins as well as drug-eluting stents has only been partially effective in reducing the rate of ISR. Since low high-density lipoprotein cholesterol (HDL-C) concentration is a pivotal cardiovascular disease risk factor, this study aimed to evaluate the compositional and functional alterations of HDL in individuals with ISR. Material and methods: This case-control study included 21 ISR, 26 non-ISR (NISR), 16 angiography-negative, and 18 healthy subjects. Serum HDL2 (d: $1.063-1.125$ $\mathrm{g} / \mathrm{ml}$ ) and HDL3 (d: 1.125-1.210 $\mathrm{g} / \mathrm{ml}$ ) subfractions were extracted from each subject using sequential ultracentrifugation. The capacity of HDL to efflux cellular cholesterol from lipid-loaded macrophages as well as to take up free cholesterol (FC) from triglyceride-rich lipoproteins (TGRLs) during lipolysis was assessed.

Results: No difference was found in the HDL2 and HDL3 content of free cholesterol and total protein among the groups. The NISR group showed lower triglyceride content in HDL2 and higher phospholipid content in HDL3 relative to healthy subjects. Strong positive correlations were found between the cholesterol efflux capacity (CEC) of HDL2 and its phospholipid content in the healthy $(r=0.50)$, angiography-negative $(r=0.55)$ and ISR $(r=0.52)$ groups. The capacity of apolipoprotein B (apoB)-depleted serum to take up free cholesterol was not different among the groups.

Conclusions: Despite some compositional alterations, the capacity of HDL to efflux cholesterol from lipid-loaded macrophages as well as to take up free cholesterol from TGRLs during lipolysis was not associated with ISR in this study.

Key words: high-density lipoprotein, cholesterol efflux capacity, reverse remnant cholesterol transport, restenosis, coronary artery disease.
Corresponding authors: Amirhossein Sahebkar Department of Medical Biotechnology School of Medicine Mashhad University of Medical Sciences

Mashhad, Iran P.O. Box: $+91779-48564$ Phone: +985138002288 Fax: +985138002287 E-mail: sahebkara@mums. ac.ir; amir_saheb2000@ yahoo.com

Anatol Kontush National Institute for Health and Medical Research (INSERM) Research Unit 1166 Faculty of Medicine Pitié-Salpêtrière Sorbonne University Paris, France E-mail: anatol.kontush@ sorbonne-universite.fr 
Shiva Ganjali, Elahe Mahdipour, Seyed Hamid Aghaee-Bakhtiari, Majid Ghayour-Mobarhan, Sara Saffar Soflaei, Maciej Banach, Anatol Kontush, Amirhossein Sahebkar

\section{Introduction}

Coronary artery disease (CAD) is a major cause of death worldwide [1]. Stent placement following percutaneous coronary intervention $(\mathrm{PCl})$ is a typical treatment for chronic heart disease (CHD), while in-stent restenosis (ISR) after $\mathrm{PCl}$ is a major clinical problem occurring in these patients [2,3]. The use of drug-eluting stents (DES) and drugs such as statins, antiplatelet agents, and calcium channel blockers has only been partially effective in reducing ISR in patients who have undergone stent implantation [2-4]. Since the number of such patients continues to increase, it is crucially important to distinguish patients who are at elevated risk of ISR. Furthermore, recognition of markers of ISR could be helpful for choosing a proper strategy in order to treat each patient [2].

Complex pathophysiological mechanisms including lipid deposition, dysfunction of endothelial, chronic vascular inflammation, and vascular smooth muscle cell proliferation causing intimal hyperplasia are involved in the progression of restenosis [5, 6]. The inverse association between the risk of restenosis following vascular intervention and high-density lipoprotein cholesterol (HDL-C) concentration, a pivotal cardiovascular disease (CVD) risk factor, has been reported $[6,7]$. However, recent studies have indicated that $\mathrm{HDL}-\mathrm{C}$ concentration alone is insufficient to correctly assess the risk of CVD [8-10]. Considering the complexity of HDL particles, alterations of their composition and function have been suggested to be more informative than HDL-C levels [11-13]. The principal anti-atherosclerotic effect of HDL is thought to be related to cellular cholesterol efflux through which accumulated cholesterol is transported to HDL or its major protein component, apolipoprotein (apo) A-I, from macrophages and arterial wall cells in an early step of reverse cholesterol transport (RCT) [6]. In addition, HDL, by virtue of cholesterol acquirement from atherogenic triglyceride-rich lipoprotein (TGRL) remnants upon their lipolysis, is involved in reverse remnant-cholesterol transport (RRT) and thereby linked to TG metabolism [10, 14].

There are limited data regarding the contribution of compositional changes and impaired function of HDL (such as its role in RCT and RRT pathways) in coronary ISR, and to our knowledge, the relationship between alterations of HDL composition and function with ISR has not previously been assessed in a case-control study. Therefore, this study was undertaken to explore the compositional characteristics of HDL as well as the capacity of HDL to efflux cholesterol from lipid-loaded macrophages and to take up free cholesterol (FC) upon lipolysis of TGRL in patients with ISR.

\section{Material and methods}

\section{Study populations}

This is a case-control study (December 2014 and April 2017), and 47 Iranian subjects (18-75 years old) were included with a history of coronary stent implantation at least 30 days earlier and thereafter due to chest pain or equivalent symptoms they were referred for re-angiography. According to the angiographic results, patients with more and less than $50 \%$ stenosis within the stent were divided into the in-stent restenosis (ISR; $N=21$ ) and non-ISR (NISR; $N=26$ ) groups, respectively. Furthermore, angiography-negative patients $(N=16)$, whose first angiographic results showed stenosis $<50 \%$, and healthy subjects $(N=$ 18) were considered as controls. The Ethics Committee of the Mashhad University of Medical Sciences gave approval of the study protocol and all participants provided written informed consent. Demographic data were collected from medical records. The exclusion criteria were positive troponin, autoimmune disease, restenosis occurring within one month after angioplasty due to thrombosis, chronic kidney disease or thrombophilia, and active cancer. A blood sample was collected in a tube with no anticoagulant just before starting the angiographic procedure. Serum was separated following centrifugation at a relative centrifugal force of 1000 (recommended by the manufacturer) for $20 \mathrm{~min}$ and then stored at $-80^{\circ} \mathrm{C}$ prior to analysis.

\section{Reagents}

Phosphate-buffered saline (PBS, 10X, including $\mathrm{KCl}(10 \mathrm{~g}), \mathrm{KH}_{2} \mathrm{PO}_{4}(10 \mathrm{~g}), \mathrm{Na}_{2} \mathrm{HPO}_{4} \times 2 \mathrm{H}_{2} \mathrm{O}$ (71.7 g), $\mathrm{NaCl}$ (400 g) (VWR, France); TopFluor cholesterol (TopF) (Avanti Polar Lipids, USA); lipoprotein lipase (LPL) from Pseudomonas sp. (Sigma, France), phosphotungstic acid (Merck, VWR), $\mathrm{MgCl}_{2}$ (VWR, France), deionized water, potassium bromide ( $\mathrm{KBr}$; 99+\%, ACS reagent, Thermo Fisher Scientific, USA), D-(+)-Glucose (Sigma, France), bovine serum albumin (BSA; Merck, USA), ([1,2-3H(N)] ( ${ }^{3} \mathrm{H}$-cholesterol) (PerkinElmer, USA), RPMI 1640 (Sigma, France), PBS (Sigma. France), fetal bovine serum (FBS; Sigma. France), phorbol 12-myristate 13-acetate (PMA; Sigma, France).

Fasting blood glucose (FBG), triglycerides (TG), total cholesterol (TC), and HDL-C were analyzed by Pars Azmoon kits (Iran) on a BT-3000 auto-analyzer (Italy). Low-density lipoprotein cholesterol (LDL-C) was calculated using the Friedewald formula. TC, phospholipid (PL), FC, and TG were mea- 
sured in HDL subfractions by microplate reader (DYNEX Technologies, USA).

\section{Preparation of density solutions}

For isolation and subfractionation of HDL density solutions ( $\mathrm{d}: 1.006,1.21$, and $1.24 \mathrm{~g} / \mathrm{ml}$ ) were prepared. At first, a solution (A; $d 1.006 \mathrm{~g} / \mathrm{ml}$ ) containing $8.76 \mathrm{~g}$ of $\mathrm{NaCl}$ in $1 \mathrm{I}$ of water and a solution of $\mathrm{NaCl} / \mathrm{KBr}(\mathrm{B} ; \mathrm{d} 1.357 \mathrm{~g} / \mathrm{ml}$ ) containing $354 \mathrm{~g} \mathrm{KBr}$ and $153 \mathrm{~g}$ of $\mathrm{NaCl}$ in 1 I of water were prepared, then, from these two, solutions of a desired density were prepared, according to the formula: $d X$ $=((V A \times d A)+(V B \times d B)) /(V A+V B)$, where $d X=$ desired density, $d A=$ density of solution $A, d B=$ density of solution $B, V A=$ volume of solution $A$, $\mathrm{VB}=$ volume of solution $\mathrm{B}$.

The densities of solutions were checked by measuring with a Digital Density Meter (DMA 45; Mettler/Paar, Austria) at $15^{\circ} \mathrm{C}[10,15]$.

\section{Isolation of HDL subfractions}

Two subfractions of HDL (HDL2 (d: 1.063-1.125 $\mathrm{g} / \mathrm{ml}$ ) and HDL3 (d: 1.125-1.210 g/ml)) were isolated by sequential ultracentrifugation from $800 \mu \mathrm{l}$ of serum [10, 16]. Separation was achieved after a three-step ultracentrifugation using a TLA120 rotor at $120,000 \mathrm{rpm}$ in an Optima MAX-TL ultracentrifuge (Beckman Coulter, USA) at $15^{\circ} \mathrm{C}$, with two $\mathrm{NaCl} / \mathrm{KBr}$ solutions (d: $1.21 \mathrm{~g} / \mathrm{ml}$ and $1.24 \mathrm{~g} /$ $\mathrm{ml})$. First, by adjusting the serum density to 1.063 $\mathrm{g} / \mathrm{ml}$ with $260 \mu \mathrm{l}$ of a $\mathrm{d}=1.24 \mathrm{~g} / \mathrm{ml}$ solution, very low-density lipoproteins (VLDL), intermediate density lipoproteins (IDL) and LDL were separated after $3 \mathrm{~h}$. Then, the density of the bottom fraction containing $\mathrm{HDL}$ was adjusted to $1.125 \mathrm{~g} / \mathrm{ml}$ with $270 \mu \mathrm{l}$ of a d: $1.24 \mathrm{~g} / \mathrm{ml}$ solution, and HDL2 was collected from the top after $4 \mathrm{~h}$ ultracentrifugation. In the final step, by adjusting the bottom density to $1.21 \mathrm{~g} / \mathrm{ml}$ with $240 \mu \mathrm{l}$ of the $\mathrm{d}: 1.24 \mathrm{~g} / \mathrm{ml}$ and $200 \mu \mathrm{l}$ of $\mathrm{d}: 1.21 \mathrm{~g} / \mathrm{ml}$ solutions, HDL3 was collected from the top after $5 \mathrm{~h}$ ultracentrifugation.

Thereafter, HDL subfractions (HDL2 and HDL3) were largely dialyzed in PBS $1 \mathrm{X}(\mathrm{pH}: 7.4)$ using SERVAPOR dialysis tubing (MWCO 12000-14000 $\mathrm{RC}, 6 \mathrm{~mm}$, Germany) in the dark at $4^{\circ} \mathrm{C}$.

\section{Compositional analysis of HDL subfractions}

HDL2 and HDL3 subfractions were assessed for the quantity of main HDL lipid ingredients, such as PL, TC, TG, and FC, via commercially enzymatic colorimetric kits (Diasys, France). Cholesteryl ester (CE) content was computed by multiplying the difference between TC and FC by 1.67 (15). Total protein (TP) was quantified in subfractions of HDL via the bicinchoninic acid (BCA) assay. Total lipoprotein mass was calculated as the sum of PL, TG, FC, CE, and TP.

\section{Cholesterol efflux assay}

The capacity of HDL subfractions in cellular cholesterol efflux (CEC) was evaluated in a human THP-1 monocytic cell system (ATCC) at $30 \mu \mathrm{g} \mathrm{HDL}$ protein $/ \mathrm{ml}$ according to the Rached et al. protocol [17]. Briefly, cells were cultured on 24-well plates (Perkin Elmer, USA) in RPMI 1640 media with FBS $(10 \%)$ and $50 \mathrm{ng} / \mathrm{ml}$ of PMA for differentiation of monocytes to macrophage-like cells. After $48 \mathrm{~h}$ of incubation, the cells were loaded with $\left[{ }^{3} \mathrm{H}\right]$ cholesterol-labeled acetylated LDL $(1 \mu \mathrm{Ci} / \mathrm{ml})$ in RGGB media containing serum-free RPMI 1640, $2 \mathrm{mM}$ glutamine, $50 \mathrm{mM}$ glucose, $0.2 \%$ BSA, and $100 \mathrm{\mu g} / \mathrm{ml}$ antibiotic for $24 \mathrm{~h}$ for equilibration of cell cholesterol pools. The efflux of cellular cholesterol to subfractions of HDL was evaluated after $4 \mathrm{~h}$ incubation of cells in serum-free media. Finally, the CEC\% was computed through the following calculation: $\mathrm{CEC} \%=($ Medium $\mathrm{cpm} /($ medium $\mathrm{cpm}$ + cell cpm) $) \times 100$.

Specific CEC\% was characterized by subtracting nonspecific CEC\% that occurs in the cells without cholesterol acceptors.

\section{ApoB precipitation}

Phosphotungstic acid/ $\mathrm{MgCl}$, precipitation was used to remove apoB-containing lipoproteins from fasted serum for the cholesterol transfer assay. Phosphotungstic acid/ $\mathrm{MgCl}_{2}(\mathrm{pH}: 6.2 ; 5 \mu \mathrm{l})$ was added to serum samples $(50 \mu \mathrm{l})$ at a ratio of $1: 10$ by volume, incubated for $10 \mathrm{~min}$ at room temperature (RT) and centrifuged at $13,000 \mathrm{rpm}$ at $4^{\circ} \mathrm{C}$ for 30 min (5415 R, Eppendorf, USA) (10).

\section{TopF transfer to HDL}

For the evaluation of HDL capacity to take up FC through LPL-mediated TGRL lipolysis, TGRLS $(\mathrm{d}<1.019 \mathrm{~g} / \mathrm{ml})$ were obtained from a reference healthy normolipidemic subject with a single-step ultracentrifugation (40,000 rpm, $\left.24 \mathrm{~h}, 15^{\circ} \mathrm{C}\right)$ and dialyzed against PBS (pH: 7.4) using a SERVAPOR dialysis tube (MWCO 12000-14000 RC, $29 \mathrm{~mm}$, Germany) at $4^{\circ} \mathrm{C}$ in the dark, and labelled with TopFluor cholesterol (TopF). For labelling, lipoprotein-deficient plasma (LPDP) was added to the TGRL at a $1 / 100$ ratio (LPDP/TGRL) by volume and thereafter was filtered using a $0.8 \mu \mathrm{m}$ filter and a chloroformic solution of TopF was added at a TopF/TGRL PL ratio of $1 / 13$ by mass; the mixture was incubated overnight at $37^{\circ} \mathrm{C}$ under gentle stirring. Then unbound fluorescent lipid was separated from labelled TGRL by filtration through a PD10 Sephadex column (GE Healthcare, Sephadex G-25M, USA). Purified labelled TGRL was assessed for TG concentration by photometry, and, to verify the labelling, TopF fluorescence was recorded at $500 / 525 \mathrm{~nm}(\mathrm{Ex} / \mathrm{Em})$ using a microplate read- 
er (Gemini, Molecular Devices, USA). To evaluate the TopF transfer, $30 \mathrm{mg} \mathrm{TG/dl}$ of TopF-labelled TGRL, Tris buffer $(0.4 \mathrm{M})(\mathrm{pH}$ : 8), final dilution of HDL (as apoB-depleted serum) 30-fold v/v, and LPL $(7600 \mathrm{U} / \mathrm{l})$ was mixed on ice and in order to start lipolysis incubated at $37^{\circ} \mathrm{C}$. After $2 \mathrm{~h}$, the reaction mixture was placed on ice and an apoB precipitant reagent (phosphotungstic acid/ $\mathrm{MgCl}_{2}$ ) was added and the mixture incubated for 10 min at RT, followed by centrifugation at $4^{\circ} \mathrm{C}$ for 10 min at a maximal speed (5415 R, Eppendorf). At the end, the extracted supernatant containing $\mathrm{HDL}$ was filtered $(0.45 \mu \mathrm{m})$ and transferred to a black microplate (Corning, USA) to read the fluorescence. The fluorescence measured in $\mathrm{HDL}$ was represented as a percentage of fluorescence in the standard sample containing TGRL (30 mg TG/dl), Tris buffer, and PBS alone.

In addition, all values were normalized to that detected in the reference serum obtained from the healthy normolipidemic control subjects [10].

\section{Statistical analysis}

For statistical analysis, SPSS software, version 11.5 (Chicago, IL, USA) was used. P-values less than 0.05 were considered statistically significant. Normal distributed variables are shown as mean \pm $\mathrm{SE}$ and differences in variables were evaluated by one-way analysis of variance (ANOVA) tests across 4 groups (healthy, angiography-negative, NISR and ISR) or a $t$-test for independent samples between 2 groups (ISR and NISR). Categorical variables are presented as percentages and between-group differences in variables were evaluated by a $\chi^{2}$ analysis or Fisher's exact test.

The association of CEC with HDL composition was evaluated using Pearson's correlation coefficients. Binary logistic regression was used to estimate the association between CEC and TopF transfer to HDL with ISR after adjustment for age, sex, DM, dyslipidemia, hypertension (HTN), stent type, hs-CRP, use of statins, aspirin and clopidogrel.

\section{Results}

\section{Baseline characteristics and patient disposition}

Of the total of 81 unrelated Iranian participants, $21,26,16$, and 18 were categorized into ISR, NISR, angiography-negative, and healthy groups, respectively (Table I). The healthy group included younger subjects than the ISR and NISR groups. The number of diabetic, dyslipidemic, and hypertensive patients revealed significant differences between the groups. Moreover, the groups were significantly different in terms of medications used such as diabetic drugs, statin, aspirin and clopidogrel. In addition, TC, HDL-C, and LDL-C were significantly higher in healthy subjects than in other groups. TG concentration was higher in the ISR group than in the angiography-negative group. Furthermore, FBS level was higher in the ISR group compared with the healthy and angiography-negative groups (Table I). Finally, the percentage of patients who had DES was significantly greater in the NISR group in comparison with the ISR group (Table II).

\section{Compositional characterization of HDL subfractions}

There were no differences in the content of FC and TP, either in HDL2 or in HDL3, among the studied groups. However, the HDL2 subfraction revealed TG enrichment in healthy subjects compared to NISR and angiography-negative patients, while its PL content was lower in the healthy group compared to ISR, NISR and angiography-negative patients. Moreover, high CE content was observed in HDL2 of healthy subjects when compared to the ISR and NISR groups (Figure 1). In addition, TG enrichment was also observed in HDL3 of healthy individuals when compared to NISR and angiography-negative patients, and the PL content of the HDL3 subfraction was statistically significantly lower in the healthy group compared to the NISR group (Figure 1).

The PL/TP ratio in HDL2 was significantly lower in healthy subjects relative to the ISR and NISR groups, while the TC/TP ratio in HDL2 was significantly higher in healthy subjects relative to the ISR group (Table I).

\section{Cellular cholesterol efflux to HDL subfractions}

There were no differences in the capacity of HDL2 and HDL3 subfractions to efflux cellular cholesterol from lipid-loaded macrophages between the studied groups (Table III). However, when CEC was normalized to HDL-C levels, significantly higher CEC/HDL-C ratios were found for both HDL2 and HDL3 in the NISR group in comparison with healthy subjects. Moreover, significantly higher HDL2 CEC/HDL-C ratios were found in the ISR and angiography-negative groups in comparison with healthy subjects (Table III). In addition, subgroup analysis revealed that subjects with diabetes in the ISR group displayed a lower HDL2 CEC/HDL-C ratio compared to those in the NISR group (Figure $2 \mathrm{C}$ ). Moreover, individuals with age above 50 had a higher CEC/HDL-C ratio of HDL2 and HDL3 in the NISR group when compared with that in the healthy group (Figure $2 \mathrm{C}$ ).

Furthermore, significant positive correlations were detected between CEC of HDL2 and its PL content in the healthy $(r=0.50)$, angiography-negative $(r=0.55)$ and ISR $(r=0.52)$ groups (Figure 3$)$ as well as in the whole study population $(r=0.48$; Figure 4). Similarly, HDL2 CEC was correlated with 
Table I. Baseline characteristics of study groups

\begin{tabular}{|c|c|c|c|c|c|}
\hline Variables & ISR $(n=21)$ & NISR $(n=26)$ & $\begin{array}{l}\text { Angiography- } \\
\text { negative } \\
(n=16)\end{array}$ & $\begin{array}{l}\text { Healthy } \\
(n=18)\end{array}$ & $P$-value \\
\hline \multicolumn{6}{|l|}{ Sex \%: } \\
\hline Male & 52.4 & 50.0 & 31.3 & 50.0 & \multirow[t]{2}{*}{0.573} \\
\hline Female & 47.6 & 50.0 & 68.8 & 50.0 & \\
\hline Age [years] & $59.5 \pm 2.6$ & $60.4 \pm 2.1$ & $54.1 \pm 2.8$ & $48.5 \pm 2.1^{\mathrm{a}, \mathrm{b}}$ & $0.003^{*}$ \\
\hline Height [cm] & $161.5 \pm 2.6$ & $162.8 \pm 2.0$ & $158.3 \pm 2.2$ & $168.1 \pm 2.2^{c}$ & $0.037^{\star}$ \\
\hline Weight [kg] & $69.9 \pm 2.8$ & $73.2 \pm 3.5$ & $66.7 \pm 3.1$ & $74.6 \pm 2.7$ & 0.341 \\
\hline BMI $\left[\mathrm{kg} / \mathrm{m}^{2}\right]$ & $27.0 \pm 0.9$ & $27.5 \pm 1.0$ & $26.8 \pm 1.0$ & $26.5 \pm 0.9$ & 0.891 \\
\hline \multicolumn{6}{|l|}{ BMI \%: } \\
\hline Normal & 33.3 & 32.0 & 37.5 & 38.9 & \multirow[t]{3}{*}{0.999} \\
\hline Overweight & 38.9 & 44.0 & 37.5 & 38.9 & \\
\hline Obese & 27.8 & 24.0 & 25.0 & 22.2 & \\
\hline Smoker \% & 5.9 & 23.1 & 14.3 & 22.2 & 0.489 \\
\hline Dyslipidemia \% & 71.4 & 46.2 & 37.5 & 0.0 & $<0.001^{*}$ \\
\hline DM \% & 61.9 & 46.2 & 12.5 & 0.0 & $<0.001^{*}$ \\
\hline HTN \% & 71.4 & 65.4 & 50.0 & 0.0 & $<0.001^{*}$ \\
\hline \multicolumn{6}{|l|}{ Drug consumption \%: } \\
\hline Statin & 100.0 & 88.0 & 56.3 & 0.0 & $<0.001^{*}$ \\
\hline Aspirin & 90.5 & 84.0 & 68.8 & 11.1 & $<0.001^{*}$ \\
\hline Clopidogrel & 68.4 & 92.0 & 12.5 & 0.0 & $<0.001^{*}$ \\
\hline Insulin & 19.0 & 19.2 & 0.0 & 0 & \multirow[t]{2}{*}{$0.001^{*}$} \\
\hline Oral diabetic drugs & 38.1 & 19.2 & 12.5 & 0 & \\
\hline $\mathrm{FBS}[\mathrm{mg} / \mathrm{dl}]$ & $155.6 \pm 16.9$ & $138.9 \pm 15.7$ & $98.2 \pm 9.1^{\text {a }}$ & $90.4 \pm 2.0^{\mathrm{a}}$ & $0.003^{*}$ \\
\hline $\mathrm{TC}[\mathrm{mg} / \mathrm{dl}]$ & $145.6 \pm 7.9$ & $124.4 \pm 8.7$ & $140.2 \pm 6.9$ & $190.7 \pm 7.2^{\mathrm{a}, \mathrm{b}, \mathrm{c}}$ & $<0.001^{*}$ \\
\hline TG [mg/dl] & $144.8 \pm 21.2$ & $97.3 \pm 11.8$ & $86.2 \pm 8.6^{a}$ & $117.8 \pm 13.5$ & $0.042^{*}$ \\
\hline $\mathrm{HDL}-\mathrm{C}[\mathrm{mg} / \mathrm{dl}]$ & $36.6 \pm 1.6$ & $34.1 \pm 2.2$ & $38.3 \pm 3.1$ & $51.1 \pm 3.0^{\mathrm{a}, \mathrm{b}, \mathrm{c}}$ & $<0.001^{*}$ \\
\hline LDL-C [mg/dl] & $81.7 \pm 6.7$ & $70.8 \pm 6.4$ & $84.7 \pm 5.9$ & $116.0 \pm 4.8^{\mathrm{a}, \mathrm{b}, \mathrm{c}}$ & $<0.001^{*}$ \\
\hline $\mathrm{SBP}[\mathrm{mm} \mathrm{Hg}]$ & $125.7 \pm 3.6$ & $121.8 \pm 2.9$ & $117.3 \pm 3.6$ & $114.4 \pm 3.6$ & 0.111 \\
\hline $\mathrm{DBP}[\mathrm{mm} \mathrm{Hg}]$ & $78.1 \pm 2.4$ & $74.8 \pm 1.6$ & $73.3 \pm 2.7$ & $75.7 \pm 2.1$ & 0.482 \\
\hline \multicolumn{6}{|l|}{ HDL2: } \\
\hline $\mathrm{PL} / \mathrm{TP}$ & $0.70 \pm 0.03$ & $0.73 \pm 0.03$ & $0.70 \pm 0.03$ & $0.60 \pm 0.02^{\mathrm{a}, \mathrm{b}}$ & $0.004^{*}$ \\
\hline $\mathrm{TC} / \mathrm{TP}$ & $0.30 \pm 0.02$ & $0.40 \pm 0.02$ & $0.40 \pm 0.02$ & $0.40 \pm 0.01^{a}$ & $0.011^{*}$ \\
\hline Total mass & $95.6 \pm 7.2$ & $91.8 \pm 6.3$ & $101.4 \pm 4.8$ & $96.6 \pm 7.2$ & 0.794 \\
\hline \multicolumn{6}{|l|}{ HDL3: } \\
\hline $\mathrm{PL} / \mathrm{TP}$ & $0.50 \pm 0.03$ & $0.50 \pm 0.02$ & $0.50 \pm 0.03$ & $0.43 \pm 0.02$ & 0.084 \\
\hline $\mathrm{TC} / \mathrm{TP}$ & $0.20 \pm 0.01$ & $0.30 \pm 0.01$ & $0.30 \pm 0.02$ & $0.30 \pm 0.02$ & 0.325 \\
\hline Total mass & $43.3 \pm 2.9$ & $46.0 \pm 2.9$ & $48.6 \pm 3.8$ & $45.3 \pm 3.3$ & 0.731 \\
\hline HDL2/HDL3 & $2.4 \pm 0.3$ & $2.1 \pm 0.2$ & $2.2 \pm 0.2$ & $2.3 \pm 0.2$ & 0.731 \\
\hline
\end{tabular}

Data are expressed as mean \pm SE or percentage; ${ }^{*}$ statistically significant $(p<0.05)$; ${ }^{a} p<0.05$ in comparison with ISR; ${ }^{b} p<0.05$ in comparison with NISR; $c p<0.05$ in comparison with angiography-negative. SBP-systolic blood pressure, DBP-diastolic blood pressure.

its FC content in the NISR group ( $r=0.5$; Figure 3). A significant positive correlation of HDL3 CEC with its FC content was also observed in the ISR $(r=0.63)$ and NISR $(r=0.627)$ groups (Figure 3$)$ as well as in the whole study population $(r=0.42$; Figure 4). Similarly, HDL3 CEC was correlated with its TG content in the angiography-negative group $(r=0.52$; Figure 3$)$. In addition, TP content of HDL2 and FC content of HDL3 showed negative and positive correlations with CEC, respectively, in the whole study population (Figure 4).

The results of binary logistic regression also failed to show any association of HDL2 and HDL3 CEC with the risk of ISR in this study (Table IV). 
Shiva Ganjali, Elahe Mahdipour, Seyed Hamid Aghaee-Bakhtiari, Majid Ghayour-Mobarhan, Sara Saffar Soflaei, Maciej Banach, Anatol Kontush, Amirhossein Sahebkar

Table II. Clinical characteristics of ISR and NISR groups

\begin{tabular}{|c|c|c|c|}
\hline Variables & $\operatorname{ISR}(n=21)$ & NISR $(n=26)$ & $P$-value \\
\hline \multicolumn{4}{|l|}{ Stent type \%: } \\
\hline Bare & 50.0 & 11.1 & \multirow[t]{2}{*}{$0.015^{*}$} \\
\hline Drug & 50.0 & 88.9 & \\
\hline \multicolumn{4}{|l|}{ Stent number \%: } \\
\hline 1 & 66.7 & 65.4 & \multirow[t]{2}{*}{0.927} \\
\hline$>1$ & 33.3 & 34.6 & \\
\hline De novo stenosis \% & 57.1 & 60.9 & 0.802 \\
\hline Duration of stent implantation [months] & $32.8 \pm 5.9$ & $22.4 \pm 5.4$ & 0.200 \\
\hline Ejection fraction (\%) & $46.2 \pm 2.9$ & $45.2 \pm 2.5$ & 0.806 \\
\hline Serum apoA-l [mg/dl] & $125.3 \pm 3.9$ & $108.4 \pm 7.2$ & 0.057 \\
\hline hs-CRP $[\mathrm{mg} / \mathrm{l}]$ & $3.90 \pm 0.80$ & $4.62 \pm 0.80$ & 0.509 \\
\hline HDL2 CEC/apoA-I & $0.030 \pm 0.01$ & $0.044 \pm 0.01$ & 0.054 \\
\hline HDL3 CEC/apoA-I & $0.04 \pm 0.01$ & $0.05 \pm 0.01$ & 0.058 \\
\hline TopF transfer/apoA-I & $0.80 \pm 0.03$ & $0.93 \pm 0.10$ & 0.136 \\
\hline
\end{tabular}

Data are expressed as mean $\pm S E$ or percentage; *statistically significant $(p<0.05)$. ApoA-I - apolipoprotein A-I, CEC - cholesterol efflux capacity, HDL - high-density lipoprotein, Hs-CRP - high-sensitivity C-reactive protein.

A

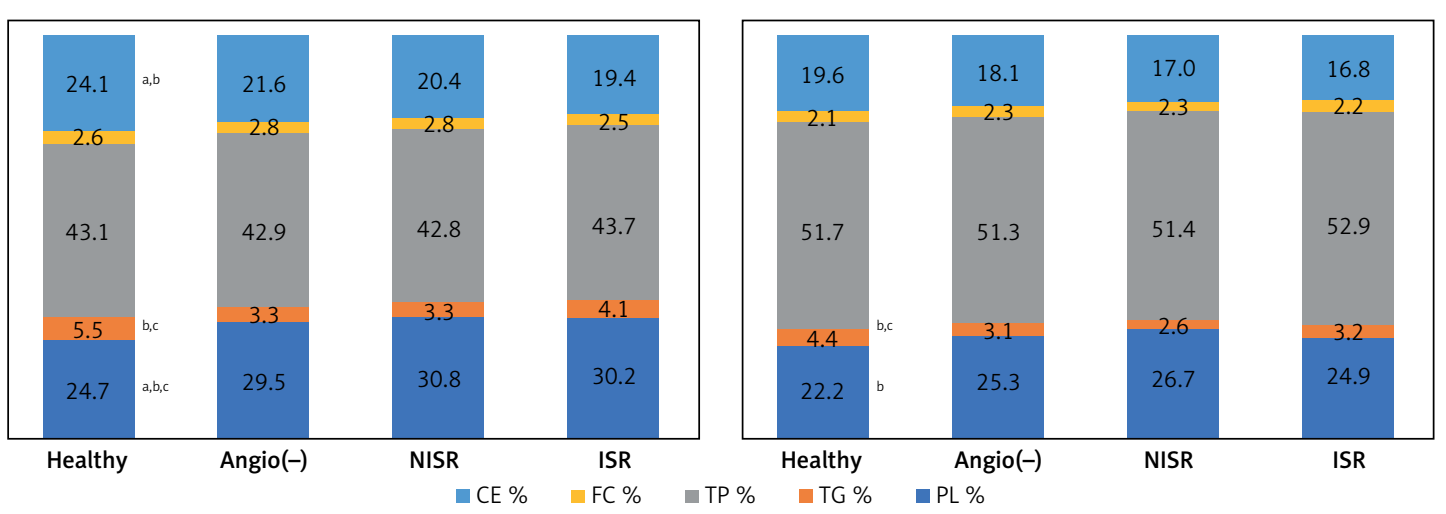

Figure 1. Comparison of $\%$ lipid and protein content of HDL subtractions between the groups: $\mathbf{A}-\mathrm{HDL} 2$ composition, B - HDL3 composition; ${ }^{a} p<0.05$ vs. ISR; ${ }^{b} p<0.05$ vs. NISR; ${ }^{c} p<0.05$ vs. angiography-negative group

Table III. Comparison of CEC and TopF transfer to HDL between groups of study

\begin{tabular}{|lccccc|}
\hline Variables & ISR $(n=21)$ & NISR $(n=26)$ & $\begin{array}{c}\text { Angiography- } \\
\text { negative } \\
(n=16)\end{array}$ & Healthy $(n=18)$ & $P$-value \\
\hline HDL2 CEC (\%) & $3.6 \pm 0.3$ & $4.5 \pm 0.4$ & $3.7 \pm 0.4$ & $3.4 \pm 0.2$ & 0.137 \\
\hline HDL3 CEC (\%) & $4.8 \pm 0.4$ & $5.2 \pm 0.4$ & $4.9 \pm 0.3$ & $4.3 \pm 0.2$ & 0.213 \\
\hline TopF transfer to HDL (\%) & $98.6 \pm 3.4$ & $99.6 \pm 6.5$ & $90.4 \pm 3.8$ & $93.9 \pm 4.6$ & 0.548 \\
\hline HDL2 CEC/HDL-C & $0.10 \pm 0.01$ & $0.13 \pm 0.01$ & $0.10 \pm 0.01$ & $0.10 \pm 0.01^{\mathrm{b}}$ & $0.002^{*}$ \\
\hline HDL3 CEC/HDL-C & $0.14 \pm 0.01$ & $0.20 \pm 0.02$ & $0.14 \pm 0.01$ & $0.10 \pm 0.01^{\mathrm{a}, \mathrm{b}, \mathrm{c}}$ & $<0.001^{*}$ \\
\hline TopF transfer/HDL-C & $2.8 \pm 0.12$ & $3.1 \pm 0.34$ & $2.6 \pm 0.2$ & $1.9 \pm 0.11^{\mathrm{a}, \mathrm{b}}$ & $0.003^{*}$ \\
\hline
\end{tabular}

Data are expressed as mean $\pm S E$; "statistically significant $(p<0.05)$; ${ }^{a}$ significant in comparison with ISR; ${ }^{b}$ significant in comparison with NISR; CEC - cholesterol efflux capacity, HDL-C - high-density lipoprotein cholesterol.

\section{TopF transfer to HDL}

No significant difference in the capacity of apoB-depleted serum to take up FC from TGRL upon LPL lipolysis was found among the studied groups. However, when TopF transfer to HDL was normalized to HDL-C levels, significantly higher TopF transfer/HDL-C ratios were observed in the ISR and NISR groups in comparison with healthy subjects (Table III). In addition, subgroup analysis 
A
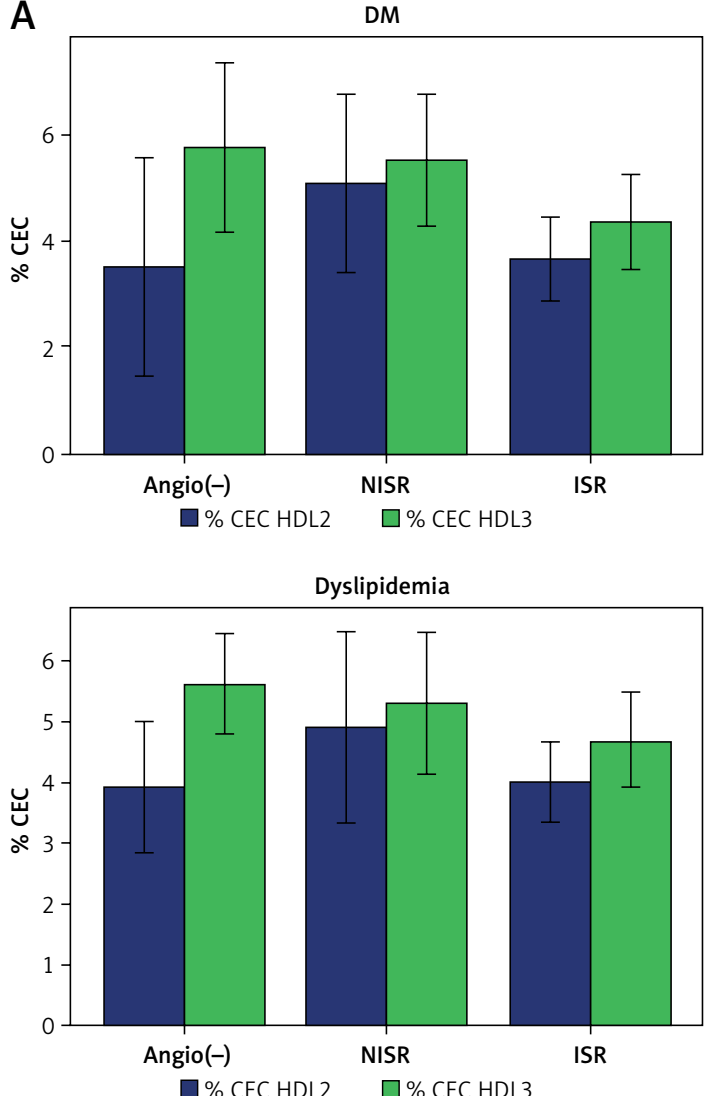

B
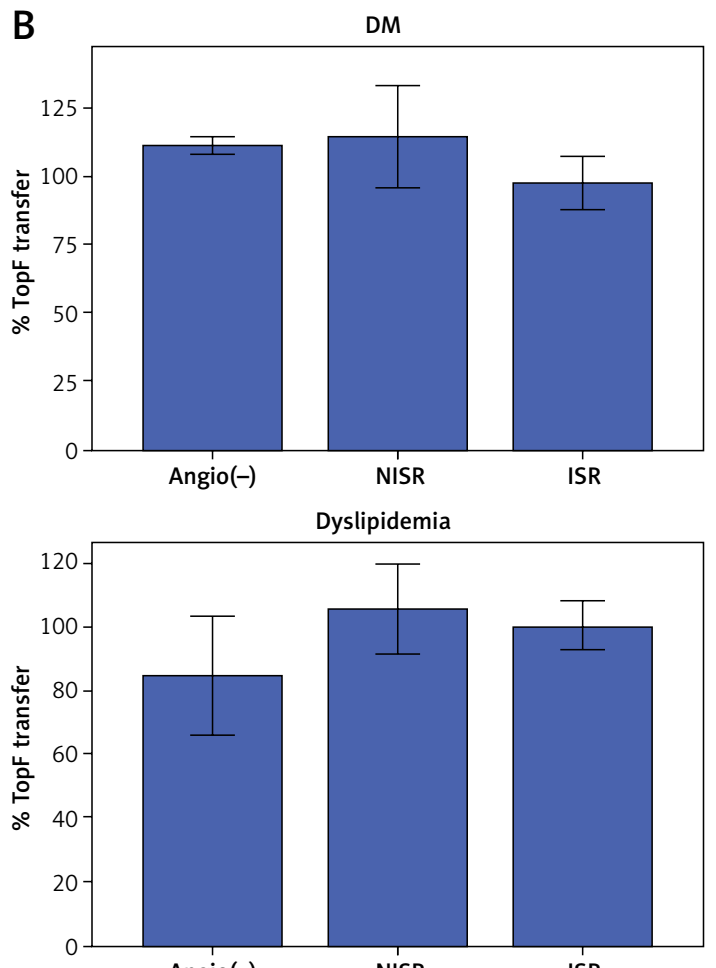

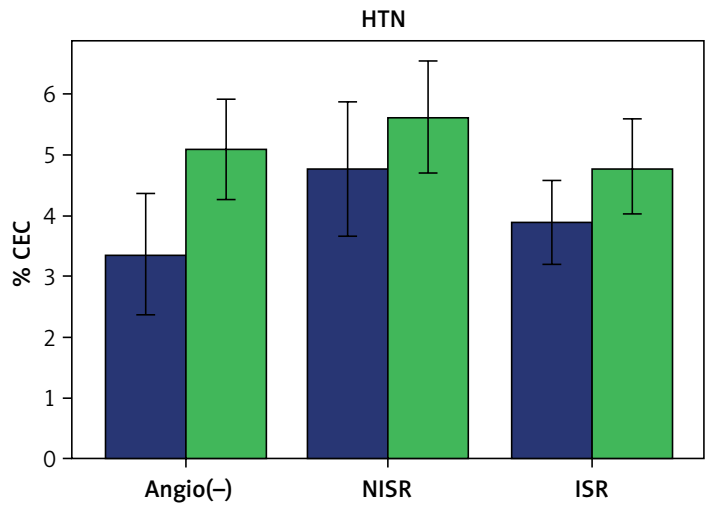

$\square \%$ CECHDL2 $\square \%$ CEC HDL3

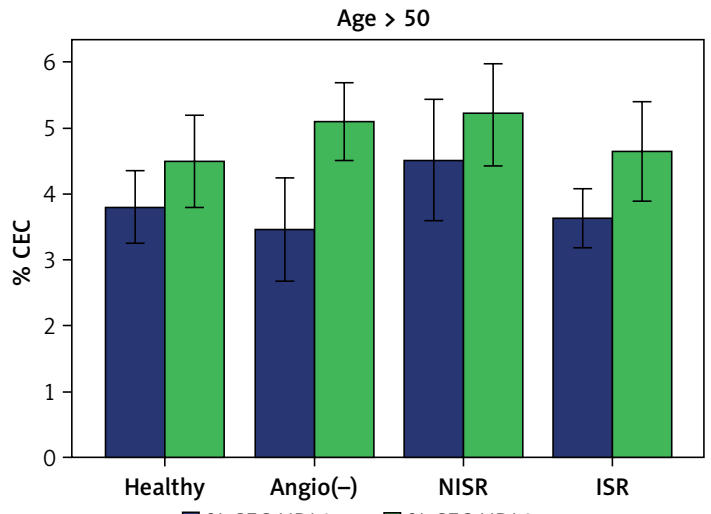

$\square \%$ CEC HDL2 $\square \%$ CEC HDL3
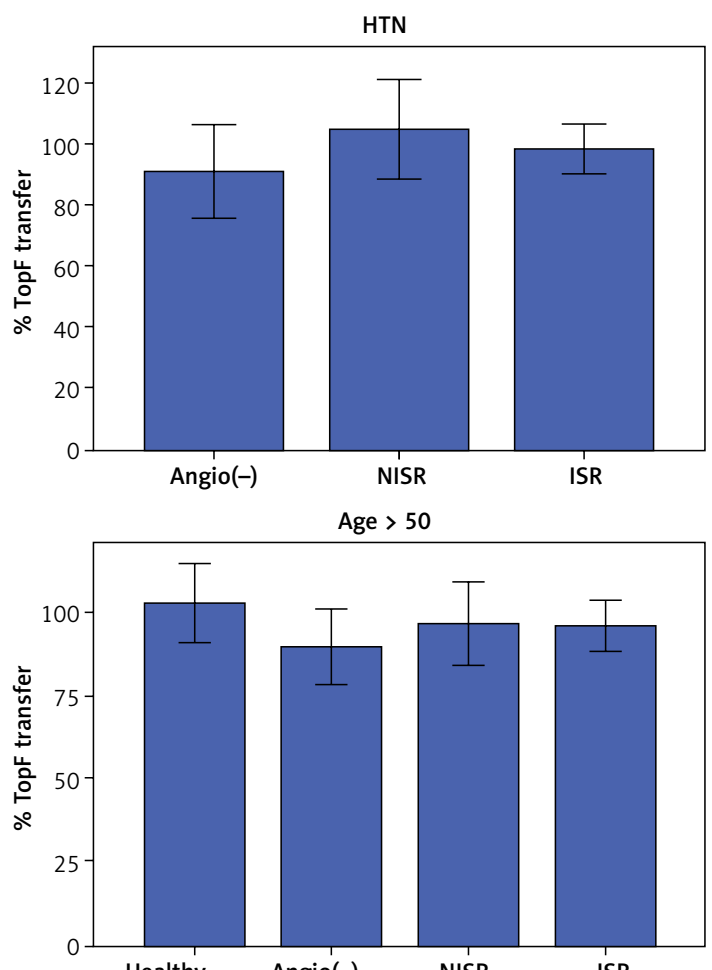

Figure 2. Comparison of CEC (A), TopF transfer (B), CEC to HDL-C ratio (C), and TopF transfer to HDL-C ratio (D) between study subgroups with diabetes, hypertension, dyslipidemia, and age $>50$ years as cardiovascular risk factors. ${ }^{*} P<0.05$ vs. ISR; ${ }^{* *} p<0.05$ vs. NISR 
Shiva Ganjali, Elahe Mahdipour, Seyed Hamid Aghaee-Bakhtiari, Majid Ghayour-Mobarhan, Sara Saffar Soflaei, Maciej Banach, Anatol Kontush, Amirhossein Sahebkar
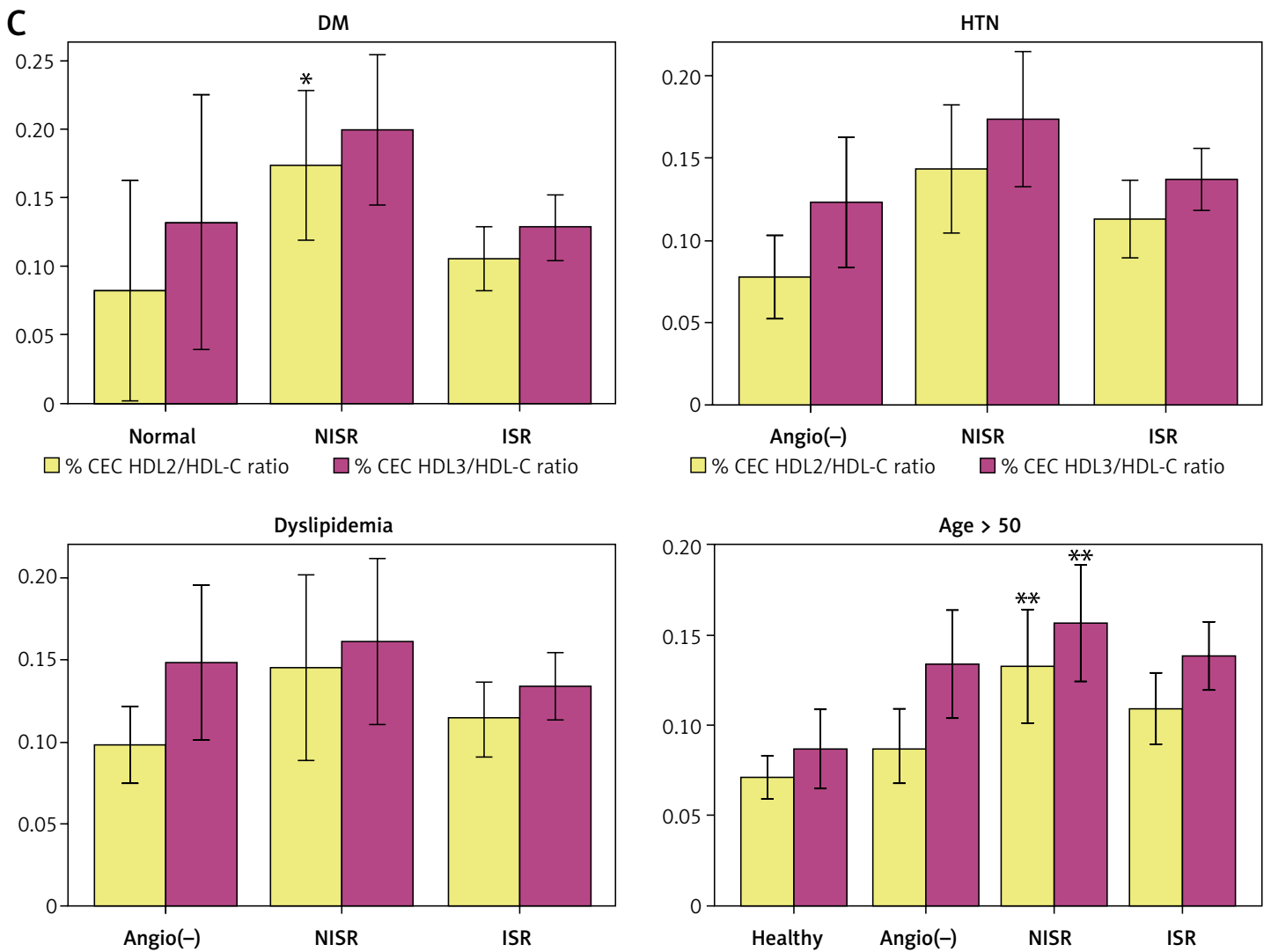

$\square \%$ CEC HDL2/HDL-C ratio $\square \%$ CEC HDL3/HDL-C ratio
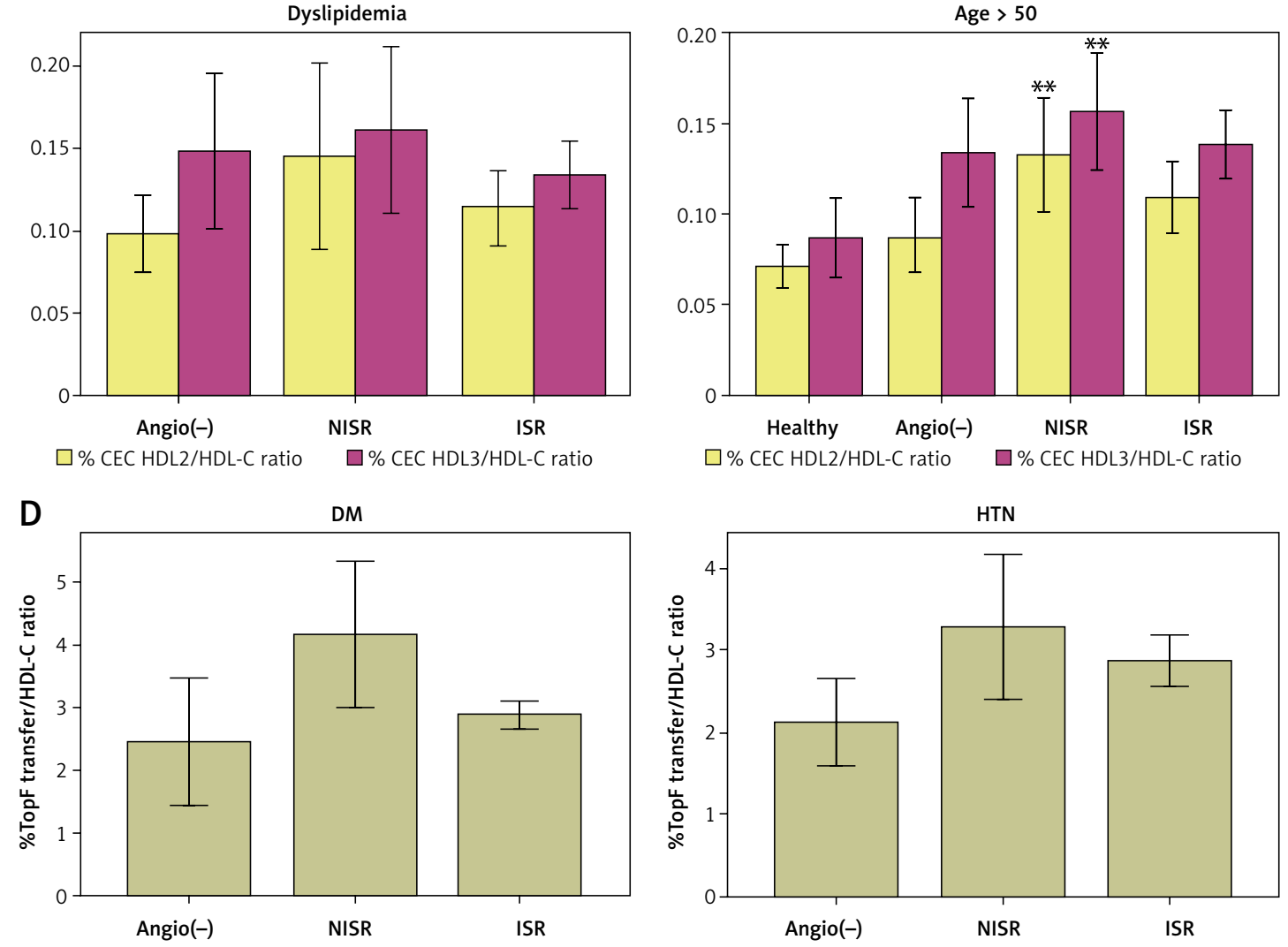

$\square \%$ CEC HDL2/HDL-C ratio $\square \%$ CEC HDL3/HDL-C ratio
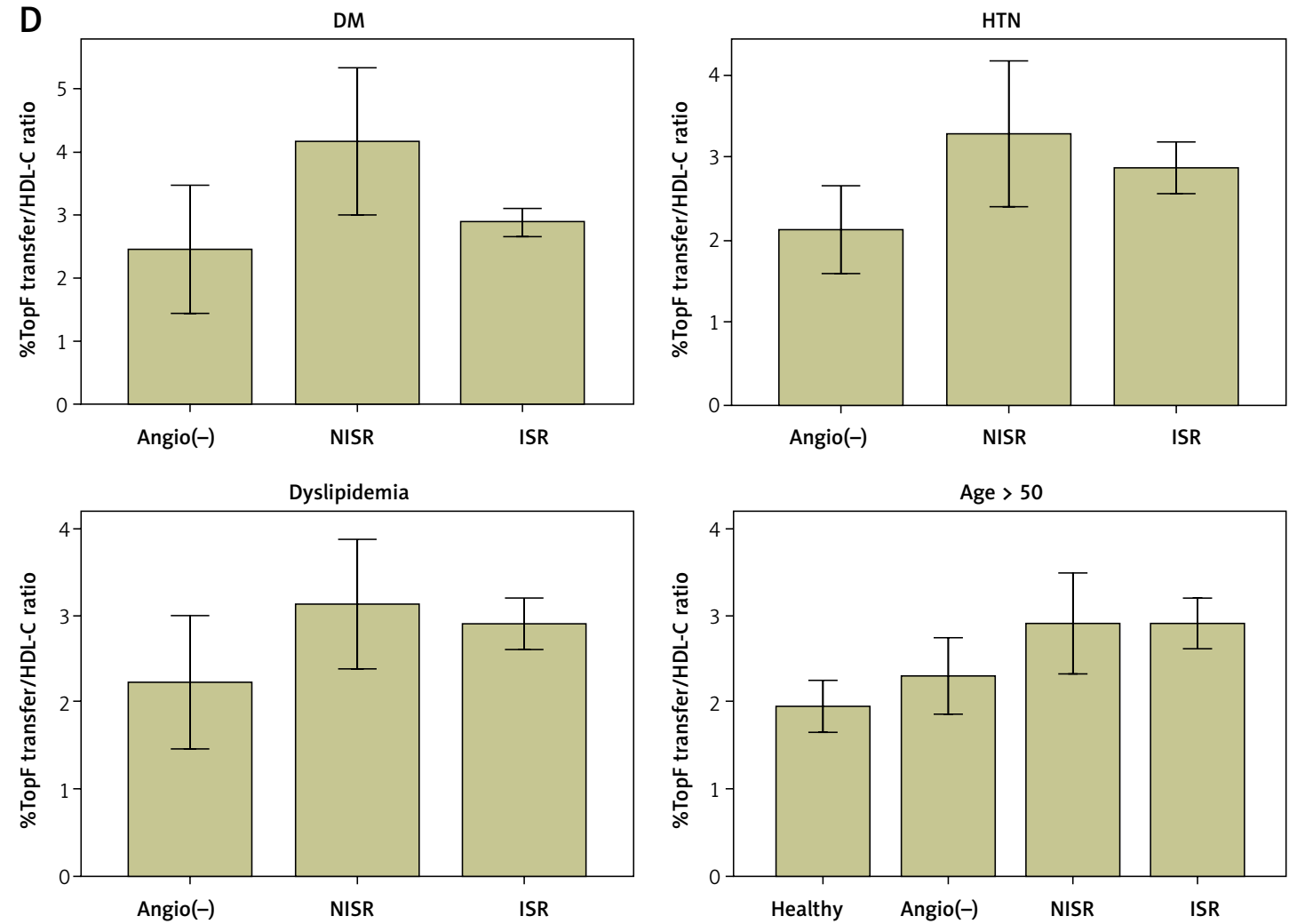

Figure 2. Cont. 

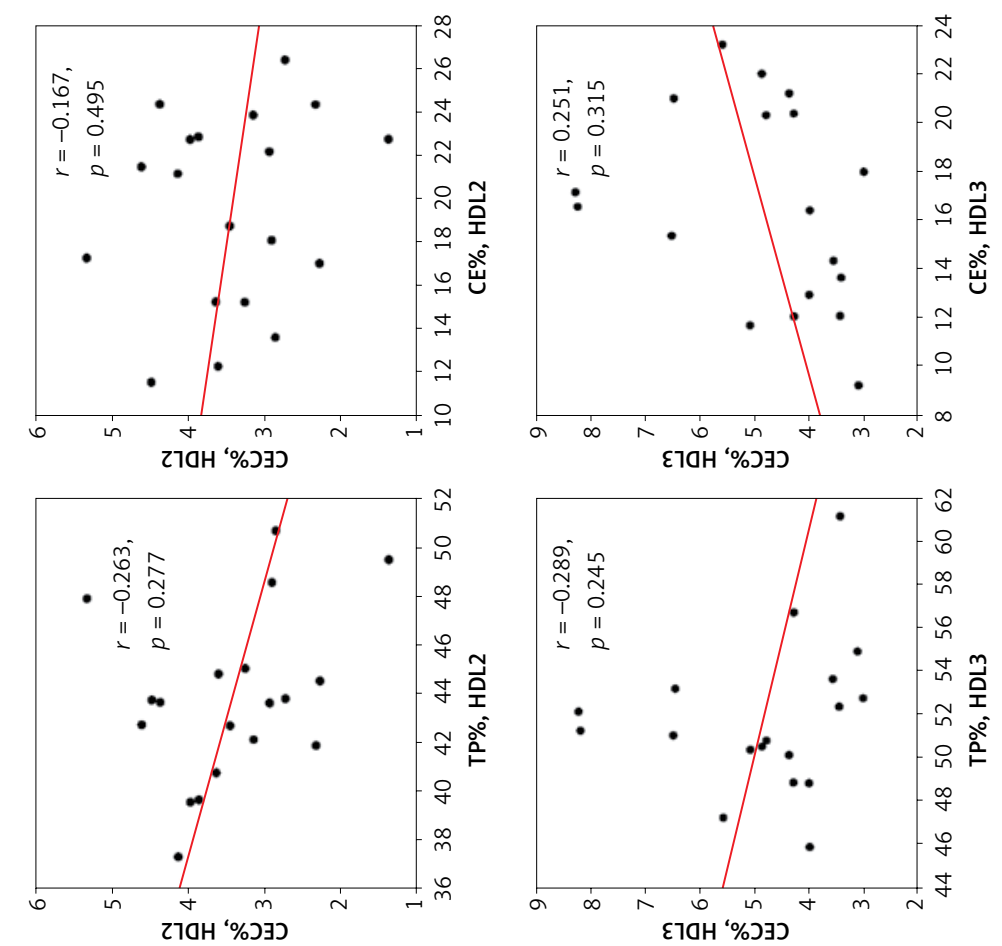

a
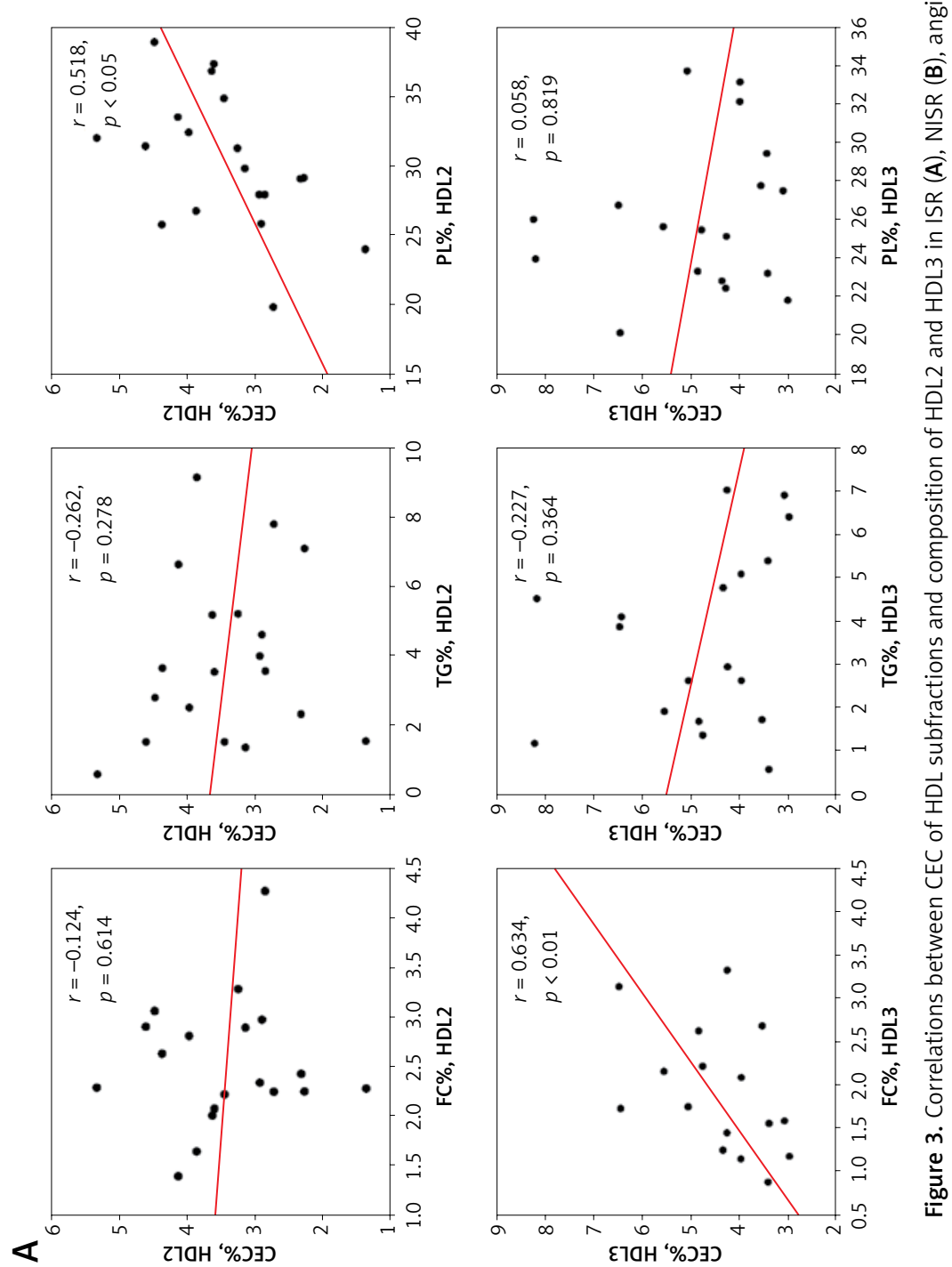
Shiva Ganjali, Elahe Mahdipour, Seyed Hamid Aghaee-Bakhtiari, Majid Ghayour-Mobarhan, Sara Saffar Soflaei, Maciej Banach, Anatol Kontush, Amirhossein Sahebkar
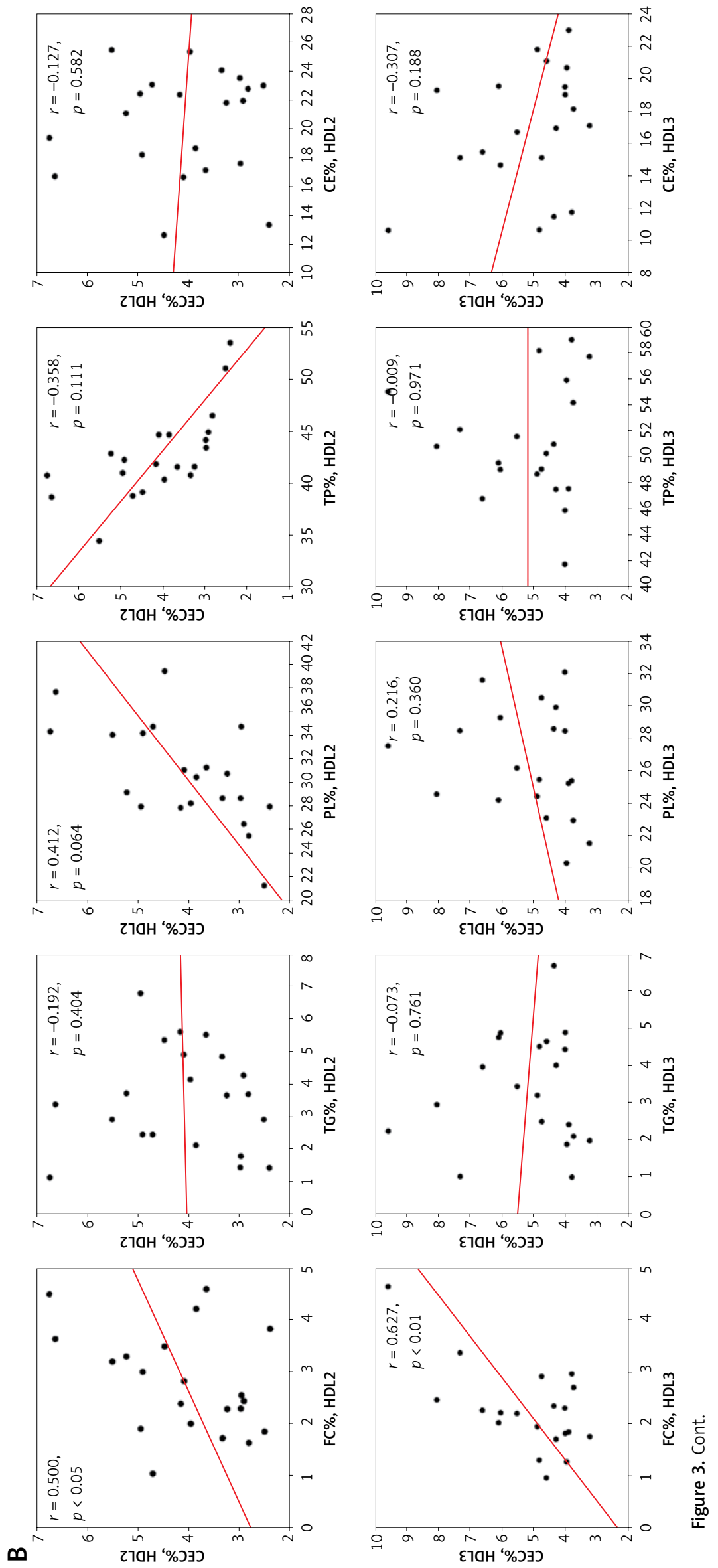

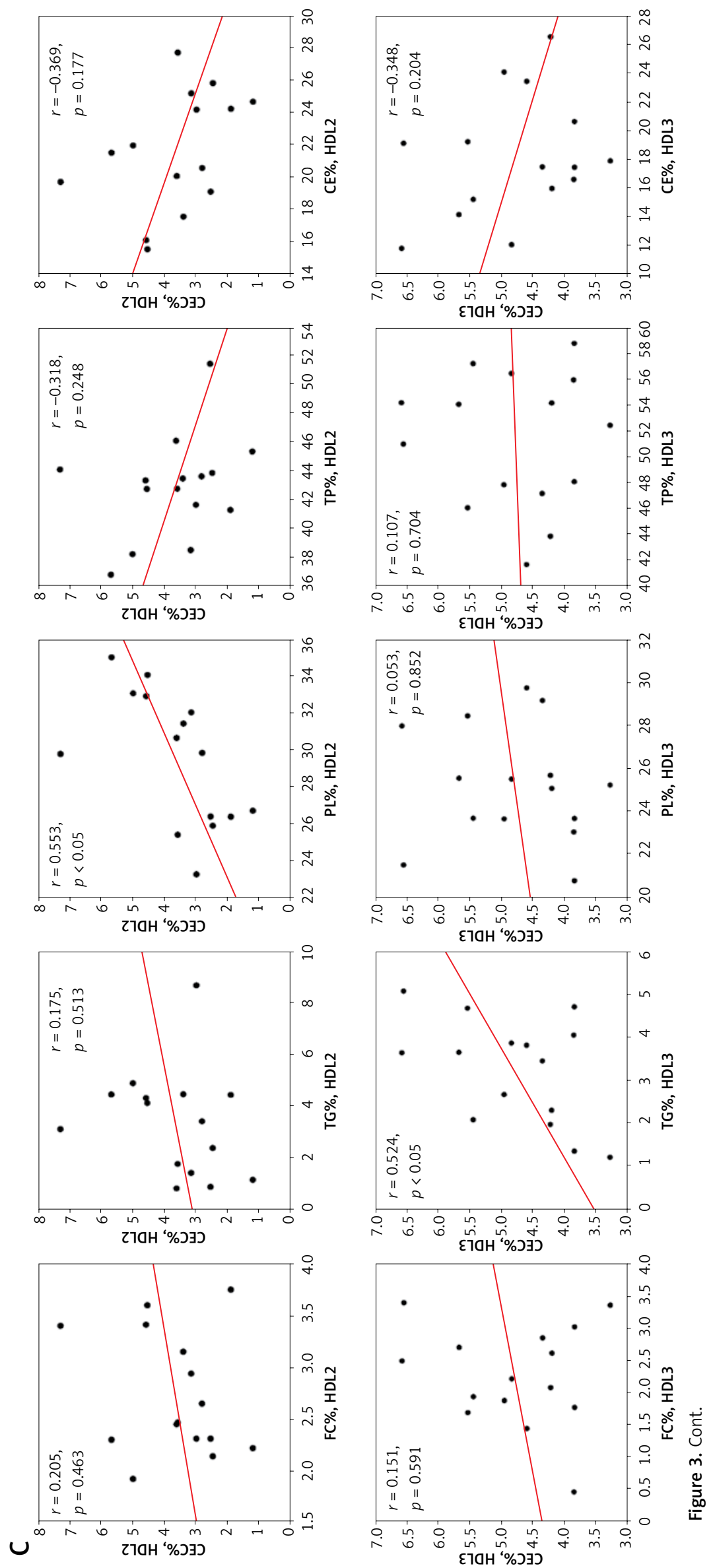
Shiva Ganjali, Elahe Mahdipour, Seyed Hamid Aghaee-Bakhtiari, Majid Ghayour-Mobarhan, Sara Saffar Soflaei, Maciej Banach, Anatol Kontush, Amirhossein Sahebkar
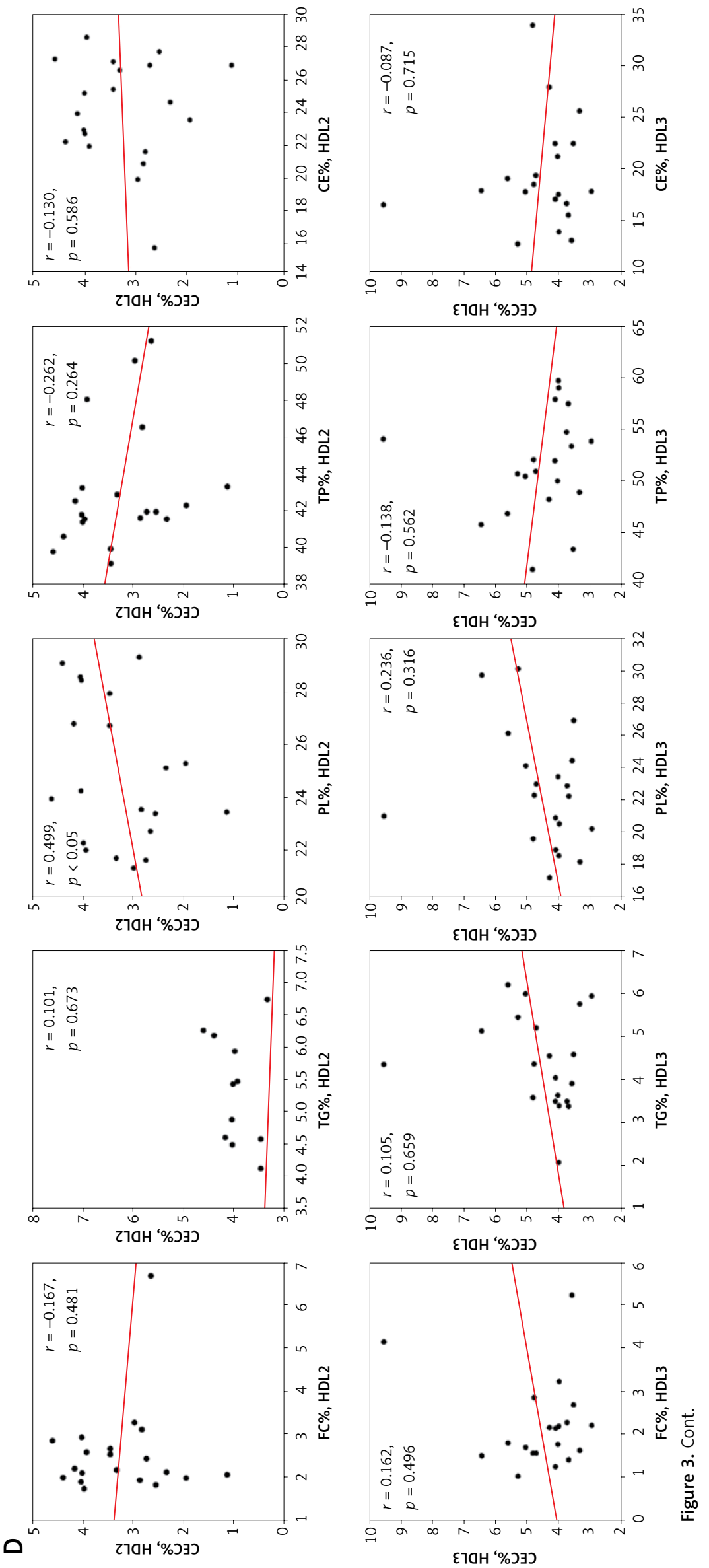

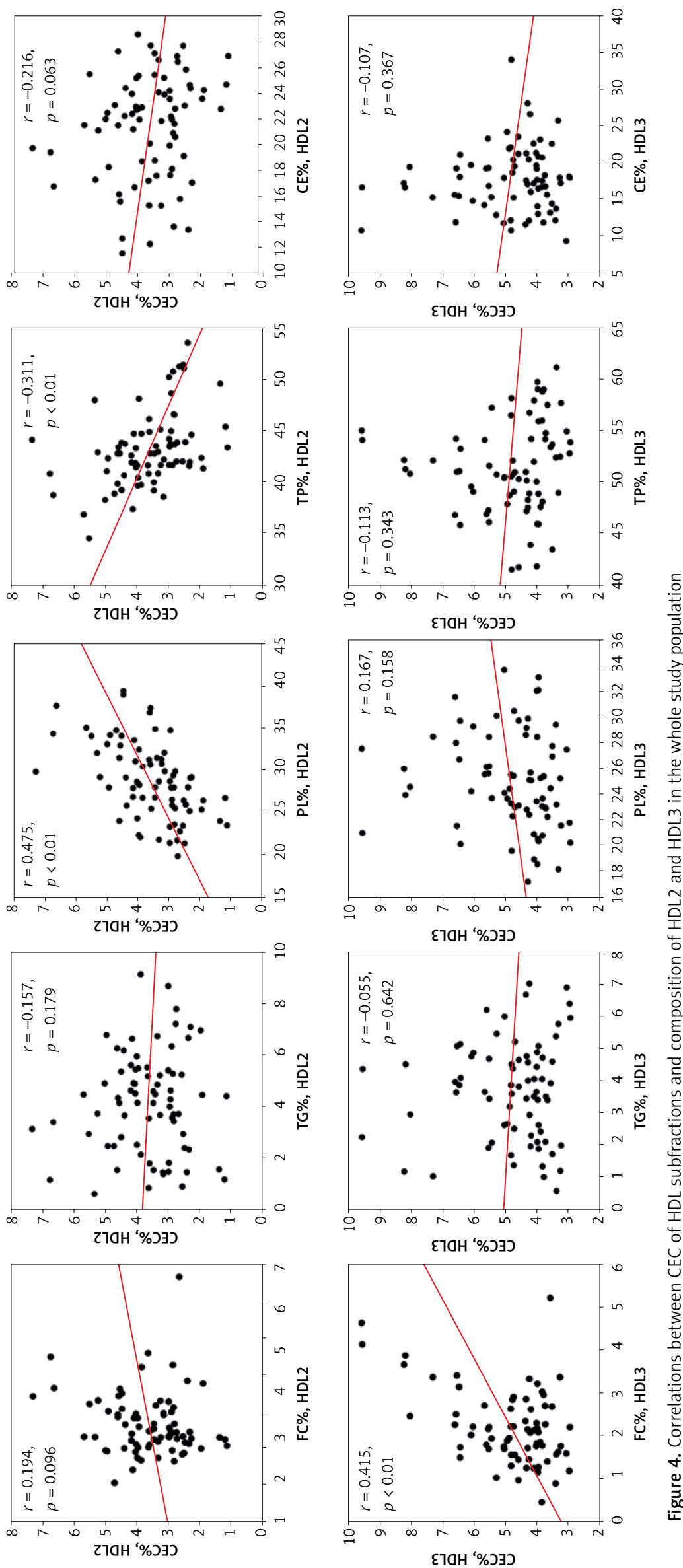
Shiva Ganjali, Elahe Mahdipour, Seyed Hamid Aghaee-Bakhtiari, Majid Ghayour-Mobarhan, Sara Saffar Soflaei, Maciej Banach, Anatol Kontush, Amirhossein Sahebkar

Table IV. Binary logistic regression for CEC and TopF transfer to HDL in relation with ISR (Ref: NISR)

\begin{tabular}{|lcccc|}
\hline Variables & \multicolumn{2}{c}{ Unadjusted } & \multicolumn{2}{c|}{ Adjusted $^{\#}$} \\
\cline { 2 - 5 } & OR $(95 \% \mathrm{Cl})$ & $\boldsymbol{P}$-value & OR (95\% Cl) & $\boldsymbol{P}_{\text {-value }}$ \\
\hline HDL2 CEC (\%) & $0.70(0.43-1.13)$ & 0.138 & $0.268(0.013-5.713)$ & 0.399 \\
\hline HDL3 CEC (\%) & $0.83(0.60-1.30)$ & 0.372 & $0.567(0.095-3.397)$ & 0.535 \\
\hline TopF transfer to HDL (\%) & $1.00(0.97-1.03)$ & 0.894 & $1.094(0.953-1.255)$ & 0.201 \\
\hline
\end{tabular}

"Adjusted for age, sex, DM, dyslipidemia, HTN, stent type, hs-CRP, use of statin, aspirin and clopidogrel.

revealed that diabetes, dyslipidemia, HTN, and age $>50$ did not influence either TopF transfer to $\mathrm{HDL}$ or TopF transfer/HDL-C ratio in the studied groups (Figure 2).

The results of binary logistic regression failed to show any association of TopF transfer to HDL with the risk of ISR in this study (Table IV).

\section{Discussion}

HDL as a protein-lipid particle has been traditionally characterized by its cholesterol concentration (HDL-C). However, the inverse association between HDL-C and CV events has been questioned by the observation of a U-shaped association in large epidemiological studies [18-21]. Hence, the use of more precise indices of $\mathrm{HDL}$ composition and function has been proposed [22].

In this case-control study, the compositional and functional alterations of HDL in patients with ISR were investigated for the first time. The results illustrated that both patients with ISR and the NISR group displayed some compositional alterations of both HDL2 and HDL3, including lower $\mathrm{CE}$ and higher PL content in HDL2 as well as lower TG content in HDL3 in comparison to healthy subjects. Moreover, the NISR group showed lower TG content of HDL2 and higher PL content of HDL3 relative to healthy subjects. A strong positive correlation between HDL2, PL\% and CEC was found in the studied groups. Nevertheless, despite the increased PL\% of HDL2 in ISR and NISR patients, the capacity of HDL2 to efflux cellular cholesterol from lipid-loaded macrophages was not statistically significantly different between the studied groups. Although a strong inverse association of CEC with the risk of incident CVD was reported previously $[23,24]$, one study reported that in a cohort of generally healthy men, CEC ability to predict CHD may not be independent of HDL-C [25], and it was found that low HDL-C $(<40 \mathrm{mg} / \mathrm{dl})$ was related to dysfunctional HDL particles [26]. Furthermore, in patients with very high HDL-C, transport of cholesterol through the RCT pathway is unlikely to be impaired [14]. Therefore, CEC was normalized with $\mathrm{HDL}-\mathrm{C}$ level (CEC/HDL-C ratio) and it was observed that the HDL2, CEC/HDL-C and HDL3 CEC/ $\mathrm{HDL}-\mathrm{C}$ ratios were significantly greater in the NISR group in comparison with healthy subjects and there were significantly higher HDL2 CEC/HDL-C ratios in the ISR and angiography-negative groups in comparison with healthy subjects, which might reflect elevated levels of $\mathrm{HDL}-\mathrm{C}$ in healthy subjects, or increased CEC in the ISR, NISR and angiography-negative groups. Li et al. also found an association between raised CEC and elevated CVD over follow-up (3 years) even after adjustment for multiple parameters, including HDL-C levels [27]. In prospective longitudinal studies in relatively healthy subjects, samples were provided prior to the CHD event and HDL values might be more functional than those obtained in cross-sectional studies, but lipid profile alterations and plaque development could influence CEC over time in longitudinal studies [25]. Hence, reverse causation can occur in cross-sectional studies. Indeed, a cross-sectional study illustrated an inverse association of HDL CEC with prevalent carotid intima-media thickness [9].

Diabetes, dyslipidemia, and hypertension are three major CVD risk factors. In this regard, subpopulation analysis revealed that individuals with diabetes in the ISR group featured a notably lower HDL2 CEC/HDL-C ratio compared to those in the NISR group. In addition, diminished HDL2 CEC/HDL-C and HDL3 CEC/HDL-C ratios were also found in healthy subjects aged over 50 relative to those in the NISR group. Posadas-Sánchez et al. reported that dyslipidemic subjects are characterized by decreased CEC in statin-treated male coronary patients [26]).

Although efflux of cholesterol from macrophages, a key step of RCT, is considered as a primary function of HDL, other functions may also play a part in cardioprotection [28]. The capacity of HDL to take up FC from TGRLs upon lipolysis in the RRT pathway is another function of HDL that was proposed to describe the U-shape relationship between HDL-C and CVD and to predict CVD better than HDL-C $[10,14]$. In this study, the rate of transfer of FC to HDL from TGRLs upon lipolysis was not statistically significantly different among the studied groups. After normalization of TopF transfer to HDL-C levels, the TopF transfer/HDL-C ratio was significantly higher in both NISR and ISR groups in comparison with healthy subjects, probably reflecting the low HDL-C in the patients. FC-enriched HDL particles reportedly possess a re- 
duced capacity to acquire FC upon lipolysis of TGRLs $[10,29]$. In this study, no difference was found in the FC content of HDL subfractions across the studied groups, which is consistent with the lack of differences in TopF transfer to HDL in the study. Moreover, both cholesteryl ester transfer protein and lecithin-cholesterol acyltransferase play important roles in cholesterol accumulation in HDL upon lipolysis [10, 30]; their activities were however not evaluated in this study.

The results of binary logistic regression failed to show any association of HDL2 and HDL3 CEC, as well as TopF transfer to HDL, with the risk of ISR in this study even after adjustment for CVD risk factors including sex, age, diabetes, hypertension, dyslipidemia, hs-CRP, and use of statin, aspirin and clopidogrel as well as stent type, which showed significant differences between ISR and NISR groups (Table IV). Cahill et al. similarly found that CEC could not predict CHD risk even after adjustment for HDL-C [25].

This study showed for the first time that the assessment of HDL function in combination with HDL-C levels and other important cardiovascular risk factors including diabetes, hypertension, and dyslipidemia could not predict ISR risk after stenting. This observation might be due to the small sample size in our study. In addition, restenosis in more than one stent and de novo stenosis in other vessels were not considered in this study. Finally, while CEC is an important index of HDL functionality, it remains unclear whether other indices of $\mathrm{HDL}$ function are associated with ISR risk.

In conclusion, the current study revealed that despite compositional alterations in HDL, HDL capacity to efflux cellular cholesterol from lipid-loaded macrophages, as well as to take up FC from TGRLs upon lipolysis, could not predict ISR risk in patients who experienced stent implantation. Nevertheless, further confirmation of these findings in future large-scale studies is warranted.

\section{Acknowledgments}

Anatol Kontush and Amirhossein Sahebkar equally contributed to this work.

This project was supported by the Mashhad University of Medical Sciences Research Council (Mashhad, Iran). We gratefully acknowledge support by a grant from the National Institute for Medical Research Development (NIMAD), Tehran, Iran (Grant no: 964334), as well as by INSERM (Paris, France) and Sorbonne University (Paris, France). The first author was also supported by the WaelAlmahmeed \& IAS research training grant.

\section{Conflict of interest}

The authors declare no conflict of interest.

\section{References}

1. Bonow RO, Mann DL, Zipes DP, Libby P. Braunwald's heart disease e-book: a textbook of cardiovascular medicine. Elsevier Health Sciences 2011.

2. Imaizumi S, Miura SI, Takata K, et al. Association between cholesterol efflux capacity and coronary restenosis after successful stent implantation. Heart Vessels 2016; 31: 1257-65.

3. Liu J, Li M, Lu H, et al. Effects of probucol on restenosis after percutaneous coronary intervention: a systematic review and meta-analysis. PLoS One 2015; 10: e0124021.

4. Baktashian M, Soflaei SS, Kosari N, et al. Association of high level of Hs-CRP with in-stent restenosis: a case-control study. Cardiovasc Revasc Med 2019; 20: 583-7.

5. Welt FG, Rogers C. Inflammation and restenosis in the stent era. Arterioscler Thromb Vasc Biol 2002; 22: 1769-76.

6. Yin K, Agrawal DK. High-density lipoprotein: a novel target for antirestenosis therapy. Clin Transl Sci 2014; 7: 500-11.

7. Pantović S, Božović D, Nikolić G, et al. Markers of inflammation and antioxidant enzyme activities in restenosis following percutaneous coronary intervention. J Serbian Chem Soc 2015; 80: 143-57.

8. Cybulska B, Kłosiewicz-Latoszek L. The HDL paradox: what does it mean and how to manage low serum HDL cholesterol level? Kardiol Pol 2014; 72: 681-6.

9. Khera AV, Cuchel M, de la Llera-Moya M, et al. Cholesterol efflux capacity, high-density lipoprotein function, and atherosclerosis. N Engl J Med 2011; 364: 127-35.

10. Feng M, Darabi M, Tubeuf E, et al. Free cholesterol transfer to high-density lipoprotein (HDL) upon triglyceride lipolysis underlies the U-shape relationship between HDL-cholesterol and cardiovascular disease. Eur J Prev Cardiol 2020; 27: 1606-16.

11. Kontush A, Chapman MJ. High-density Lipoproteins: Structure, Metabolism, Function and Therapeutics. John Wiley \& Sons 2011.

12. Bacchetti T, Ferretti G, Carbone F, et al. Dysfunctional high-density lipoprotein: the role of myeloperoxidase and paraoxonase-1. Curr Med Chem 2021; 28: 2842-50.

13. Ganjali S, Watts GF, Banach M, Reiner Ž, Nachtigal P, Sahebkar A. The Yin and Yang of high-density lipoprotein and atherosclerotic cardiovascular disease: focusing on functionality and cholesterol efflux to reframe the HDL hypothesis. Curr Med Chem 2021; 28: 6066-81.

14. Kontush A. HDL and reverse remnant-cholesterol transport (RRT): relevance to cardiovascular disease. Trends Mol Med 2020; 26: 1086-100.

15. McPherson PA, Young IS, McKibben B, McEneny J. High density lipoprotein subfractions: isolation, composition, and their duplicitous role in oxidation. J Lipid Res 2007; 48: 86-95.

16. Rosenson RS, Brewer Jr HB, Chapman MJ, et al. HDL measures, particle heterogeneity, proposed nomenclature, and relation to atherosclerotic cardiovascular events. Clin Chem 2011; 57: 392-410.

17. Rached F, Santos RD, Camont L, et al. Defective functionality of HDL particles in familial apoA-I deficiency: relevance of alterations in HDL lipidome and proteome. J Lipid Res 2014; 55: 2509-20.

18. Ko DT, Alter DA, Guo H, et al. High-density lipoprotein cholesterol and cause-specific mortality in individuals without previous cardiovascular conditions. J Am Coll Cardiol 2016; 68: 2073. 
Shiva Ganjali, Elahe Mahdipour, Seyed Hamid Aghaee-Bakhtiari, Majid Ghayour-Mobarhan, Sara Saffar Soflaei, Maciej Banach, Anatol Kontush, Amirhossein Sahebkar

19. Bowe B, Xie Y, Xian H, Balasubramanian S, Zayed MA, AlAly Z. High density lipoprotein cholesterol and the risk of all-cause mortality among US veterans. Clin J Am Soc Nephrol 2016; 11: 1784-93.

20. Madsen CM, Varbo A, Nordestgaard BG. Extreme high high-density lipoprotein cholesterol is paradoxically associated with high mortality in men and women: two prospective cohort studies. Eur Heart J 2017; 38: 2478-86.

21. Hirata A, Sugiyama D, Watanabe $M$, et al. Association of extremely high levels of high-density lipoprotein cholesterol with cardiovascular mortality in a pooled analysis of 9 cohort studies including 43,407 individuals: The EPOCH-JAPAN study. J Clin Lipidol 2018; 12: 674-84.e5.

22. Riggs KA, Rohatgi A. HDL and reverse cholesterol transport biomarkers. Methodist Debakey Cardiovasc I 2019; 15: 39-46.

23. Saleheen D, Scott R, Javad S, et al. Association of HDL cholesterol efflux capacity with incident coronary heart disease events: a prospective case-control study. Lancet Diabetes Endocrinol 2015; 3: 507-13.

24. Rohatgi A, Khera A, Berry JD, et al. HDL cholesterol efflux capacity and incident cardiovascular events. N Engl J Med 2014; 371: 2383-93.

25. Cahill LE, Sacks FM, Rimm EB, Jensen MK. Cholesterol efflux capacity, HDL cholesterol, and risk of coronary heart disease: a nested case-control study in men. J Lipid Res 2019; 60: 1457-64.

26. Posadas-Sánchez R, Posadas-Romero C, Mendoza-Pérez E, et al. Cholesterol efflux and metabolic abnormalities associated with low high-density-lipoprotein-cholesterol and high triglycerides in statin-treated coronary men with low-density lipoprotein-cholesterol $<70 \mathrm{mg} /$ dl. Am J Cardiol 2012; 109: 636-41.

27. Li XM, Tang WH, Mosior MK, et al. Paradoxical association of enhanced cholesterol efflux with increased incident cardiovascular risks. Arterioscler Thromb Vasc Biol 2013; 33: 1696-705.

28. Rosenson RS, Brewer Jr HB, Davidson WS, et al. Cholesterol efflux and atheroprotection: advancing the concept of reverse cholesterol transport. Circulation 2012; 125: 1905-19.

29. Camont L, Lhomme M, Rached F, et al. Small, dense high-density lipoprotein-3 particles are enriched in negatively charged phospholipids: relevance to cellular cholesterol efflux, antioxidative, antithrombotic, anti-inflammatory, and antiapoptotic functionalities. Arterioscler Thromb Vasc Biol 2013; 33: 2715-23.

30. Schwartz CC, VandenBroek JM, Cooper PS. Lipoprotein cholesteryl ester production, transfer, and output in vivo in humans. J Lipid Res 2004; 45: 1594-607. 